\title{
Differentiating effects of socio-economic factors on relative weight and nutritional status in Polish schoolchildren across intergenerational changes
}

\author{
Natalia Nowak-Szczepanska* ${ }^{\oplus}$, Aleksandra Gomula and Slawomir Koziel \\ Department of Anthropology, Hirszfeld Institute of Immunology and Experimental Therapy, Polish Academy of Sciences \\ in Wroclaw, 50-449 Wroclaw, Poland
}

Submitted 11 October 2019: Final revision received 10 April 2020: Accepted 4 May 2020: First published online 14 July 2020

\begin{abstract}
Objective: The aim of this study was an assessment of the effects of urbanisation level, family size and parental education on body mass index (BMI) and mid-upper arm circumference (MUAC) among Polish schoolchildren in cross-sectional surveys conducted between 1966 and 2012.

Design: The analysis involved schoolchildren measured in four Polish Anthropological Surveys (1966, 1978, 1988 and 2012). Socio-economic factors involved: urbanisation level (city, town and village), family size (one child, two children, three children, four or more children), and father's and mother's education (lower and higher education).

Setting: Regions in Poland - cities: Warsaw, Lodz and Wroclaw; towns: Bystrzyca Klodzka, Pinczow, Siemiatycze, Wolsztyn and their rural surroundings.

Participants: A total sample consisted of 63757 children (31 774 boys and 31983 girls) aged 7-18 years.

Results: Between 1966 and 1988, both BMI and MUAC had significantly higher values in children from cities, in families with one child and with higher parental education $(P<0.05)$. However, MUAC revealed significant differences between particular socio-economic groups more frequently than BMI. In 2012, urbanisation level and parental education ceased to show a differentiating effect on both indicators, while family size remained a significant social factor for both measures (BMI: $P<0.05$; MUAC: $P<0.01$ ).

Conclusions: Since MUAC reflected socio-economic differences more frequently than BMI, it could be a more sensitive and reliable anthropometric measure revealing the effects of socio-economic factors on children's nutritional status.
\end{abstract}

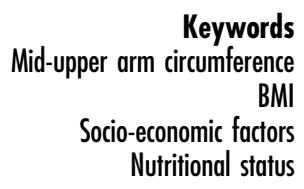

Socio-economic conditions in a given population involve issues such as national income, social inequalities, level of health care, economic and technological development that all together are affecting individual socio-economic status (SES) $)^{(1)}$. Individual living conditions usually differ concerning the urbanisation level, family size or parental education. In epidemiological auxology, one of the most acknowledged theories is Tanner's idea that the growth of children is a mirror of the socio-economic conditions ${ }^{(2)}$. However, it should be emphasised that the process of human growth has a high level of complexity and may be an effect of different, multiple factors ${ }^{(3-5)}$. Nevertheless, relative weight and nutritional status, to some extent differently than human stature ${ }^{(6)}$, are most directly related to the influence of food resources and socio-economic conditions providing appropriate access to them, as well as the adequate quality of these products.

Many anthropometric traits, such as body height, body weight or body mass index $(\mathrm{BMI}=$ weight $[\mathrm{kg}] /$ height $[\mathrm{m}]^{2}$ ), are not only influenced by intergenerational changes, defined as secular trends, but also may be affected by rapid, significant changes of socio-economic factors. Throughout history, socio-political transformations, especially from communism to capitalism and towards a well-developed form of democracy, have been observed in many countries, leading to changing socio-economic conditions that affect individual living standards ${ }^{(7,8)}$. These changes often generated nutritional transitions involving easier access to high-energy food products and different dietary habits, as well as decreased physical activity that, in total, has 
led to the increase of relative weight within a population of children $^{(9,10)}$.

Although a positive trend in relative weight may be observed across many countries, it should be noted that the urbanisation level still influences the nutritional and relative weight status of children. Among developing countries, in cities, compared with villages, children usually have a higher relative weight not only because of better living conditions and higher material status, but also due to a lower level of physical activity and the dominance of a sedentary lifestyle ${ }^{(11-13)}$. In developed countries, where an urban population, apart from better living conditions, also has a higher level of nutritional awareness and healthy lifestyle, there is a lower level of excess body weight than in rural settlements, where people have a lower SES, but currently easier access to unhealthy, high-energy food products and more sedentary activities ${ }^{(14-17)}$. Also, a recent worldwide review has shown that the main drivers of the global obesity epidemic in adults are increases in BMI in rural areas ${ }^{(18)}$.

Family size has a direct effect on food distribution within a family, resulting from a simple relationship: a higher number of children implies a smaller portion of food per person. Therefore, children from large families usually have a lower relative weight than children from families with only one child ${ }^{(19)}$. This association also has a negative epidemiological effect: children from families with one child may be at greater risk of being overweight or obese ${ }^{(19,20)}$. However, some research from Israel has shown that, particularly, parental involvement and the environment at home have a significant impact on children's eating behaviours and physical activity, and less obesogenic factors were met in families with fewer children ${ }^{(21)}$.

One of the most significant socio-economic factors affecting children's nutritional and relative weight status is parental education. Moreover, some researchers ${ }^{(22)}$ state that parental education is the best indicator showing an association between SES and excess weight in children. Higher parental education determines a lower level of overweight among children, particularly in developed societies $^{(19,22)}$. Some studies summarise that the father's education is more often related to his job prospects and providing resources for the family, while the mother's education is related to her awareness of a healthy lifestyle that directly affects the choice of quality of food and dietary habits within a given family ${ }^{(23)}$. However, it should also be noted that maternal education has a greater influence on children's relative weight than paternal education, since mothers usually spend more time with children and more strongly affect their dietary habits than fathers ${ }^{(22)}$.

BMI is a worldwide known index, commonly used for evaluation of the prevalence of overweight and obesity $^{(24)}$. Nevertheless, BMI has also become a controversial anthropometric indicator because it does not differentiate particular types of tissues within a whole-body mass, for example, muscle and adipose tissue ${ }^{(25)}$. Although mid- upper arm circumference (MUAC) is a simple anthropometric measure based on arm circumference, this indicator is highly associated with the content of adipose tissue, particularly among children ${ }^{(26)}$, while BMI shows only a moderate, but still significant, correlation with body fat during childhood ${ }^{(27)}$. Both BMI and MUAC consist of muscle and adipose tissue; however, BMI also includes visceral and extremity fat, organ size and the effects of body proportions. Moreover, it should be noted that during children's growth, weight is not proportional to height squared as well as body proportions and body fat change inconsistently with BMI; thus, this measure should be used with caution in studies on children and adolescents ${ }^{(25)}$. At the same time, several researchers have stated that MUAC is a useful and reliable biological indicator of both underweight, overweight and obesity, also among children and adolescents $^{(28-31)}$. Many studies have shown how different socio-economic conditions affect children's relative weight calculated by $\mathrm{BMI}^{(6,32,33)}$. Up to date, however, there is a lack of research comparing both these anthropometric indicators in the context of the impact of living conditions on children's relative weight and nutritional status. Therefore, the aim of this paper is the evaluation of the different effects of socio-economic factors (urbanisation, family size and parental education) on two anthropometric measures of relative weight and nutritional status, BMI and MUAC, during the broader context of intergenerational changes between 1966 and 2012 in Polish schoolchildren. Our research hypothesis is that MUAC may be a more sensitive indicator of different socio-economic factors compared with BMI. Historical background, with its socio-economic fluctuations, includes a long period of communism, before the socio-political transformation towards democracy and time of a well-developed form of a democratic society.

\section{Methods}

\section{Participants and data collection}

This investigation involved a total sample of 63757 Polish schoolchildren (31 774 boys and 31983 girls) aged 718 years. Children were examined in four subsequent cross-sectional surveys, named Polish Anthropological Surveys, conducted in 1966 ( $n 18$ 713), 1978 ( $n 19749)$, 1988 ( $n 19299)$ and $2012(n 5996)^{(10,34)}$.

Survey settlements involved schools located in cities ( $22.88 \%$ of a studied sample), towns (38.40\% of a studied sample) and villages (38.72\% of a studied sample) in different parts of Poland. Data collection was performed during a multi-stage procedure of sampling as follows: regions within Poland, the cities, towns and villages in the regions, the schools within the locations, the required number of children within the schools ${ }^{(10)}$. Survey in 1966 was nationwide (covering all contemporary districts); however, due to the high costs of this type of study, next 
Table 1 Results of the third-order interaction effects of ANOVA (without second-order interaction effects due to sufficient readability) with effect sizes measured by eta square $\left(\eta^{2}\right)$ for Z-BMI and Z-MUAC as dependent variables and sex, Survey, socio-economic factors (urbanisation, family size, mother's education and father's education) as independent variables for Polish schoolchildren measured in 1966, 1978, 1988 and 2012

\begin{tabular}{|c|c|c|c|c|c|c|}
\hline & \multicolumn{3}{|c|}{ Z-BMI } & \multicolumn{3}{|c|}{ Z-MUAC } \\
\hline & $F$ & $P$ & $\eta^{2}$ & $F$ & $P$ & $\eta^{2}$ \\
\hline Sex $\times$ Survey $\times$ urbanisation & 5.40 & $<0.001$ & 0.0005 & $9 \cdot 40$ & $<0.001$ & 0.0009 \\
\hline Sex & 6.96 & $<0.01$ & 0.0001 & 235.99 & $<0.001$ & 0.0037 \\
\hline Survey & 339.94 & $<0.001$ & 0.0159 & 963.51 & $<0.001$ & 0.0439 \\
\hline Urbanisation & 86.45 & $<0.001$ & 0.0027 & 273.97 & $<0.001$ & 0.0086 \\
\hline Sex $\times$ Survey $\times$ family size & $2 \cdot 29$ & $<0.05$ & 0.0003 & $2 \cdot 88$ & $<0.01$ & 0.0004 \\
\hline Sex & 4.79 & $<0.05$ & $<0.0001$ & $235 \cdot 30$ & $<0.001$ & 0.0038 \\
\hline Survey & 307.61 & $<0.001$ & 0.0143 & $898 \cdot 19$ & $<0.001$ & 0.0406 \\
\hline Family size & 81.64 & $<0.001$ & 0.0038 & $238 \cdot 19$ & $<0.001$ & 0.0111 \\
\hline Sex $\times$ Survey $\times$ father's education & $7 \cdot 30$ & $<0.001$ & 0.0003 & $6 \cdot 28$ & $<0.001$ & 0.0003 \\
\hline Sex & 13.90 & $<0.001$ & 0.0002 & $217 \cdot 40$ & $<0.001$ & 0.0035 \\
\hline Survey & 416.03 & $<0.001$ & 0.0199 & 1105.42 & $<0.001$ & 0.0513 \\
\hline Father's education & 41.79 & $<0.001$ & 0.0007 & $229 \cdot 13$ & $<0.001$ & 0.0037 \\
\hline Sex $\times$ Survey $\times$ mother's education & 3.41 & $<0.05$ & 0.0002 & 2.74 & $<0.05$ & 0.0001 \\
\hline Sex & 14.58 & $<0.001$ & 0.0002 & $189 \cdot 40$ & $<0.001$ & 0.0030 \\
\hline Survey & 320.41 & $<0.001$ & 0.0150 & 828.29 & $<0.001$ & 0.0380 \\
\hline Mother's education & 67.03 & $<0.001$ & 0.0011 & 341.54 & $<0.001$ & 0.0054 \\
\hline
\end{tabular}

Surveys involved three cities (Warsaw, Lodz and Wroclaw) and four towns (Bystrzyca Klodzka, Pinczow, Siemiatycze and Wolsztyn) with the villages around their county districts (finally, all analyses covered the same research areas). During each Survey, similar demographic and social characteristics were collected and anthropometric measurements were taken by the trained anthropological staff. All measurements were performed according to the standard measurement protocol described by Martin and Saller ${ }^{(35)}$. Height and MUAC were measured to the nearest $0 \cdot 1 \mathrm{~cm}$, while body mass was assessed to the nearest $0.1 \mathrm{~kg}$, using, respectively, an anthropometer, anthropometric tape and weight scales. During these measurements, children were examined wearing light clothing without shoes.

\section{Statistical analysis}

BMI was assessed as weight (in $\mathrm{kg}$ ) divided by height (in meters squared) and standardised for age (Z-BMI) according to LMS parameters, separately for boys and girls, referring to the LMS method (Lambda for the skew, Mu for the median, and Sigma for the generalised CV) developed by Cole et al. (36) MUAC was also standardised for age (Z-MUAC) using LMS parameters, separately for both sexes, referring to Mramba et al. ${ }^{(37)}$ The LMS method allows one to take into account the effect of age as a covariate that is associated with changing distribution of a given measurement during the growth of children ${ }^{(38)}$. Socio-economic factors assessed in this study involved the following: urbanisation level, family size as well as mother's and father's education. The level of urbanisation included three categories: cities (more than 100000 inhabitants), towns (up to 100000 inhabitants) and villages (rural settlements). Family size was assessed with respect to four categories: families with only one child, with two children, with three children, and with four or more children. Parental education was defined in four levels, from the lowest to the highest: elementary, trade, secondary and university; however, due to the limited number of data in some Surveys (parents with university education in 1966 and parents with elementary education in 2012), this variable was divided into two categories: higher education (university and secondary) and lower education (elementary and trade). Statistical analyses involved multivariate ANOVA for $Z$-BMI and Z-MUAC as dependent variables, and the year of Survey, sex, socio-economic factor as independent variables. The interaction effect between the independent variables was analysed with respect to its influence on dependent variables (Table 1). For post hoc comparisons, Tukey's HSD (honestly significant difference) test for unequal sample size was implemented (assumed a significance level of $P<0 \cdot 05$ ). Eta squared values $\left(\eta^{2}\right)$ were assessed as the measures of effect size for sex, year of Survey and socio-economic factors on standardised values of BMI and MUAC (Table 1). All analyses were done using Statistica 13.1 $1^{(39)}$.

\section{Results}

\section{Urbanisation level}

Within each category of the urbanisation level, both $Z$-BMI and $Z$-MUAC significantly increased between 1966 and 2012 for boys and girls $(P<0.001$; Table 1, Fig. 1; see online supplementary material). However, between 1966 and 1978, only $Z$-MUAC increased significantly in cities, towns and villages for both sexes $(P<0 \cdot 001$, except for girls in towns), while regarding Z-BMI, there were almost 
Socio-economic factors and nutritional status
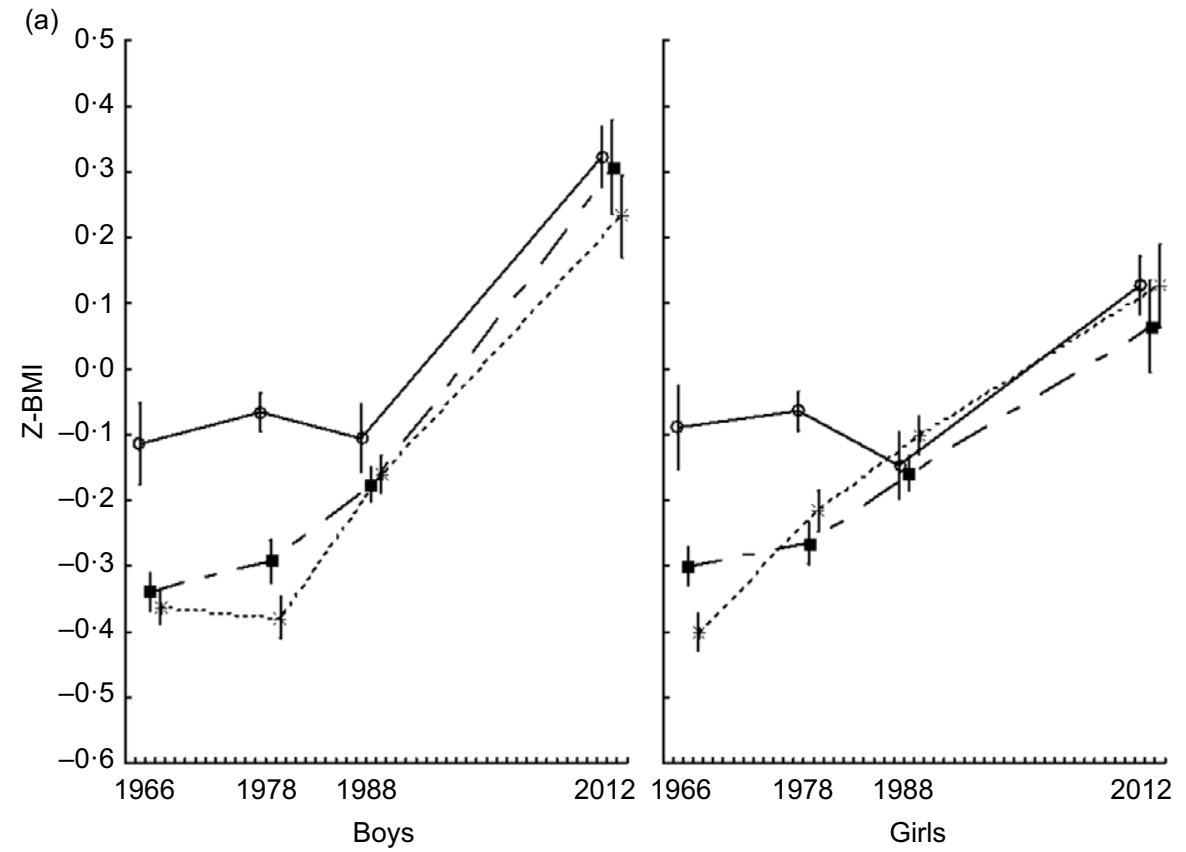

(b)
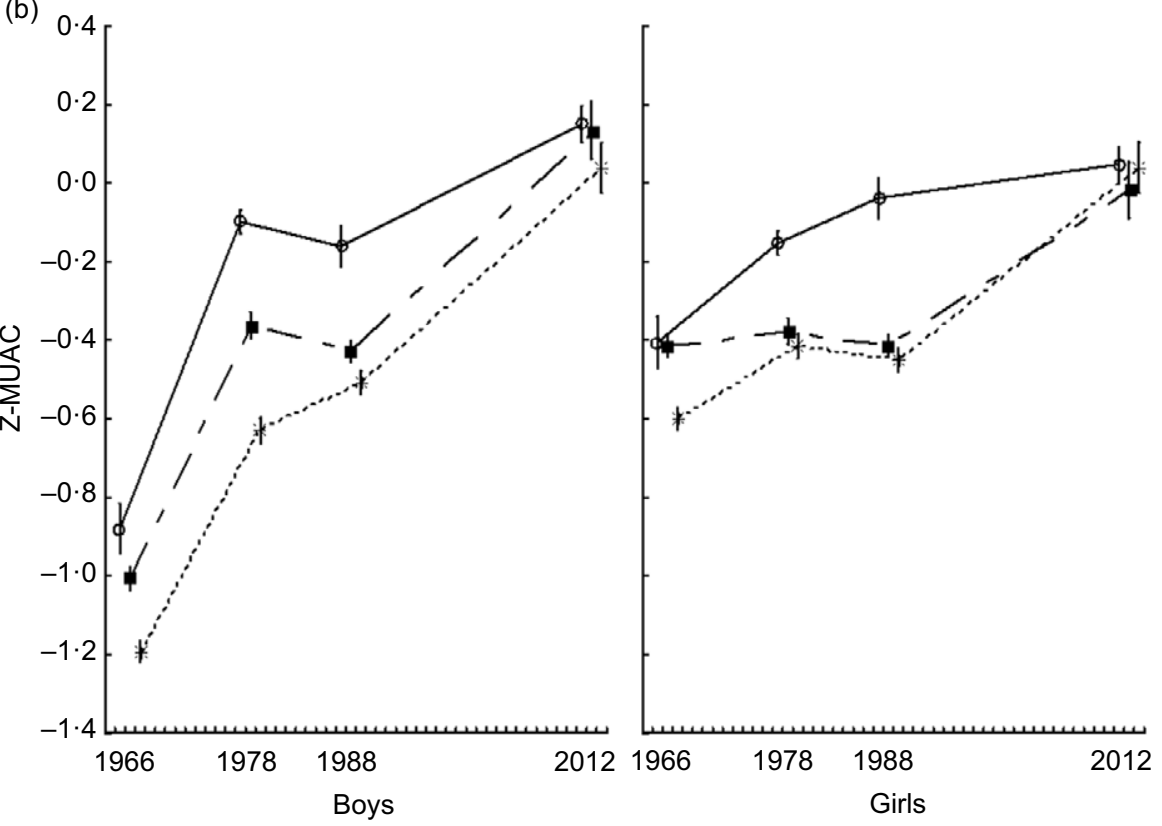

Fig. 1 (a) BMI standardised for age (Z-BMI) and (b) mid-upper arm circumference standardised for age (Z-MUAC) in boys and girls between 1966 and 2012 for different categories of urbanisation (vertical lines indicate $95 \% \mathrm{Cl}$ for the mean values). $\mp$, City; $\equiv$, town; 玉, village

no significant changes during these years $(P>0 \cdot 05$, compare: see online supplementary material). Between 1978 and 1988, almost no significant changes were noted for both variables. After 1988, significant increases in both $Z$-BMI and $Z$-MUAC were observed in almost all categories $(P<0 \cdot 001)$.

$Z$-BMI was higher among boys and girls from cities (compared with towns and villages) only in 1966 and $1978(P<0 \cdot 01$, see online supplementary materials). In most cases, $Z$-MUAC differed significantly with respect to the urbanisation level during the Surveys in 1966, 1978 and 1988 in favour of children from cities, followed by those from towns and villages (at least $P<0.05$; see online supplementary materials). In 2012, no significant differences were noted in $Z$-MUAC among boys and girls from different categories of urbanisation $(P>0.05)$.

\section{Family size}

Z-BMI and Z-MUAC significantly increased between 1966 and 2012 in each category of family size in both sexes $(P<0.01$ and $P<0.001$, respectively; Table 1, Fig. 2; see 
(a)

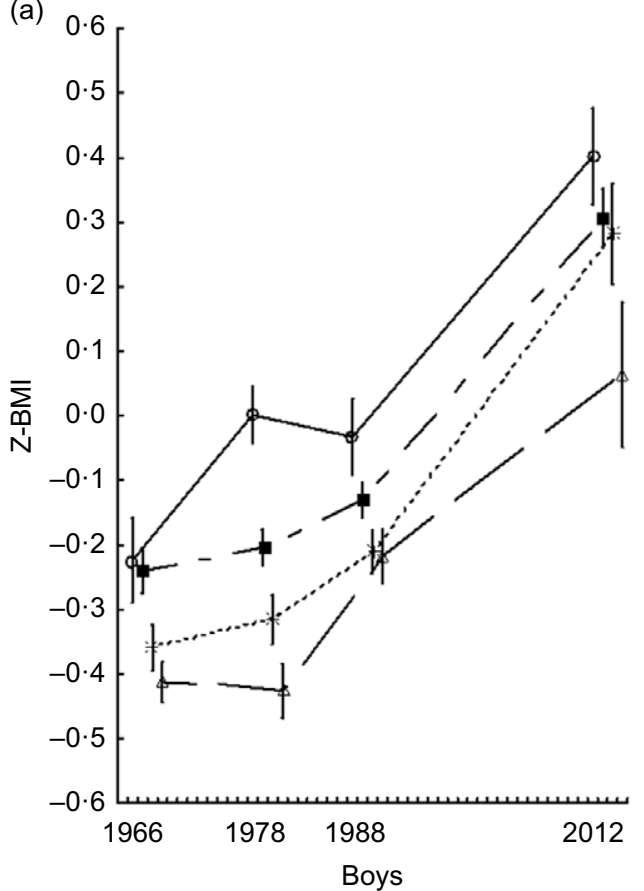

(b)

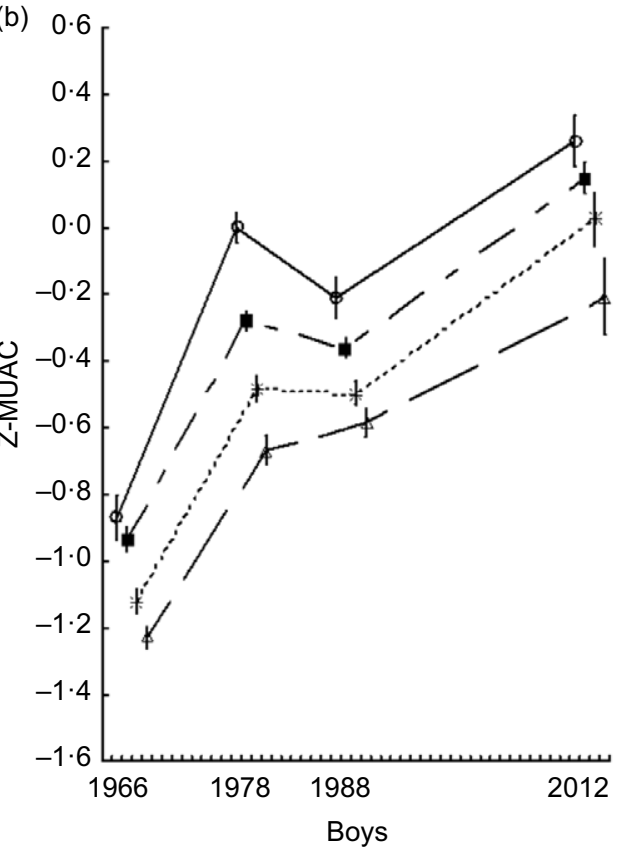

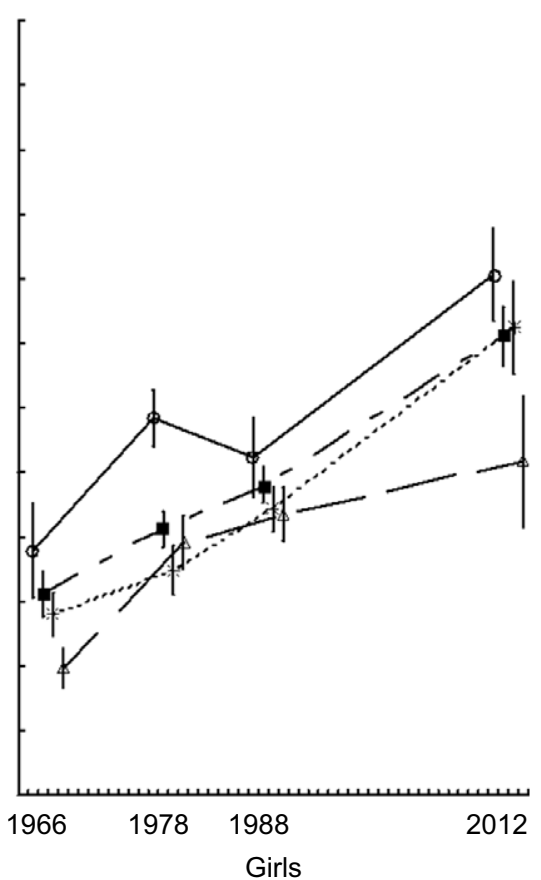

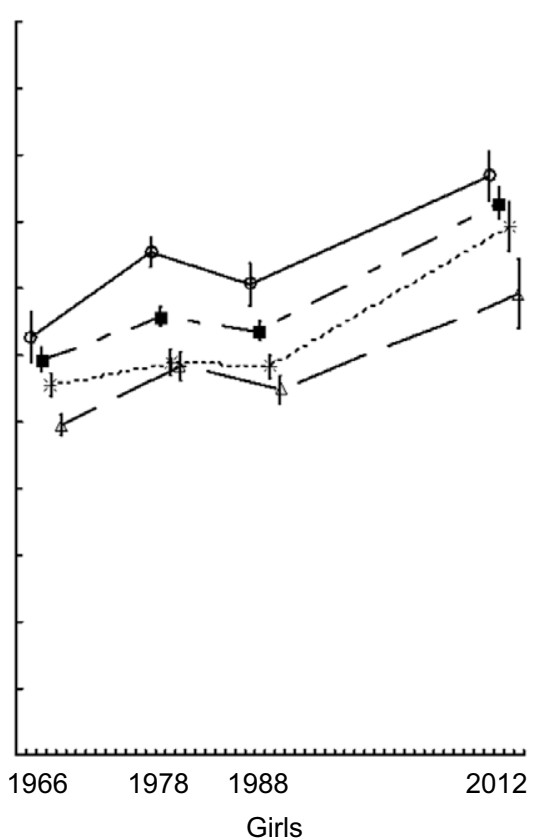

Fig. 2 (a) BMl standardised for age (Z-BMI) and (b) mid-upper arm circumference standardised for age (Z-MUAC) in boys and girls between 1966 and 2012 for different categories of family size (vertical lines indicate $95 \% \mathrm{Cl}$ for the mean values). 玉, One child; ㅍ, two children; 王, three children; 王, four or more children

online supplementary material). For $Z$-BMI, significant increases between 1966 and 1978 were observed only in a few categories, between 1978 and 1988 almost no significant changes were noted, while between 1988 and 2012, a significant increase was observed almost in all groups for both sexes $(P<0.001$; Fig. 2 ; see online supplementary material). Z-MUAC increased significantly between successive Surveys almost in all categories of family size, except for the period between the years 1978 and 1988, where no changes or significant decrease was noted (at least $P<0.001$; Fig. 2 ; see online supplementary material).

Regarding Z-BMI, most frequently, during the most years of Surveys, significant differences between particular categories of family size were observed with respect to the extreme categories, that is, families with one child (higher 

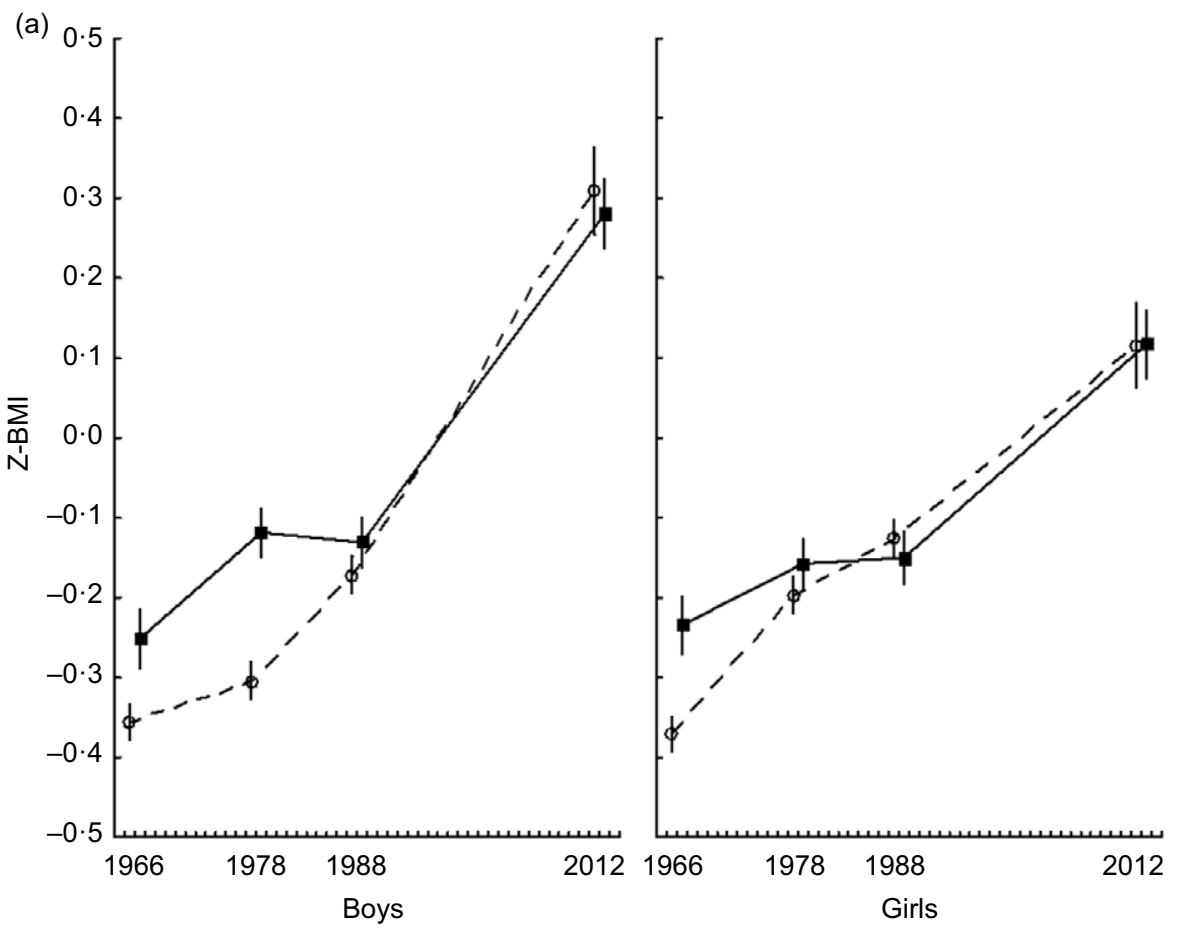

(b)
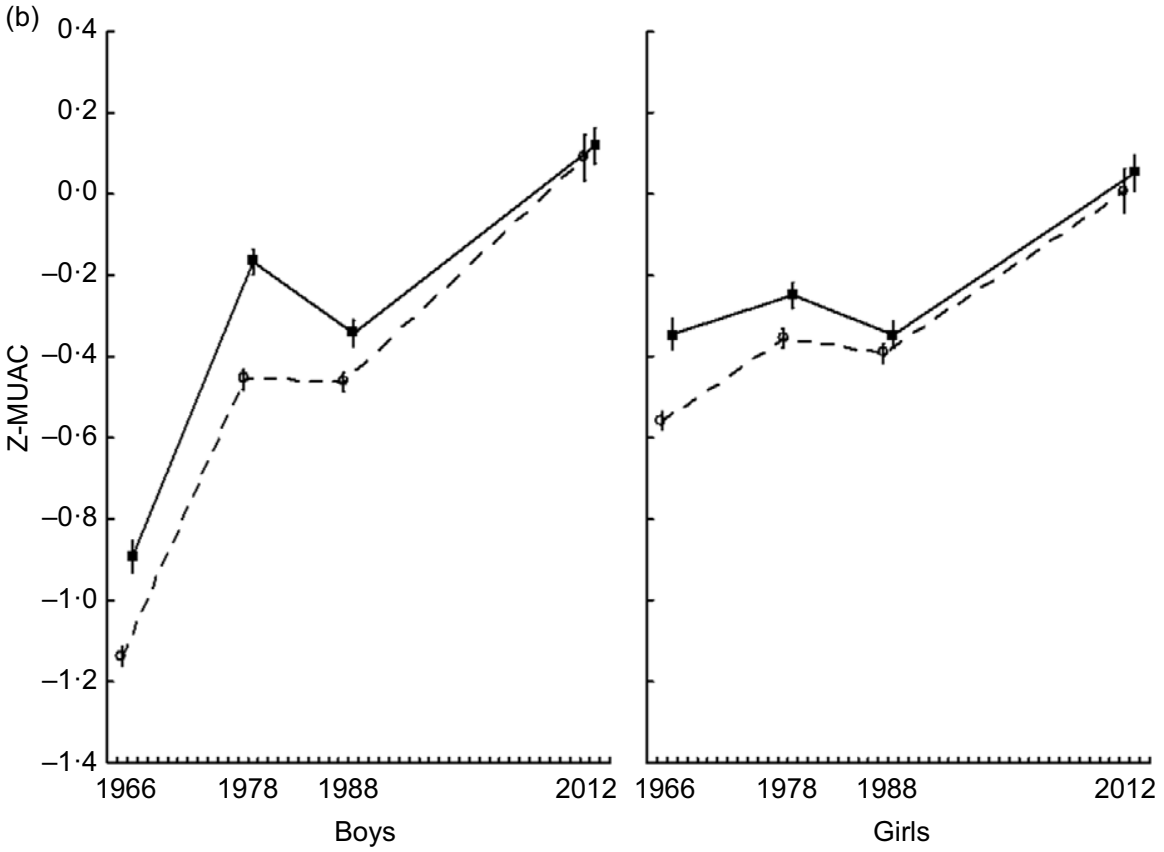

Fig. 3 (a) BMI standardised for age (Z-BMI) and (b) mid-upper arm circumference standardised for age (Z-MUAC) in boys and girls between 1966 and 2012 for different categories of father's education (vertical lines indicate $95 \% \mathrm{Cl}$ for the

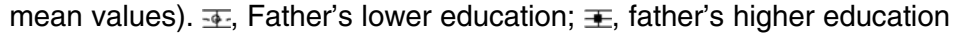

$Z$-BMI) $v$. families with four or more children (lower $Z$-BMI) (at least $P<0 \cdot 05$; except only for girls in 1988 , where no significant differences were noted), while the differences between the subsequent categories of family size were usually not prominent or non-significant (except for 1978; see online supplementary material). With respect to $Z$-MUAC, this variable was significantly differentiated by family size across all Surveys in both boys and girls: children from families with one child had the highest value of arm circumference referring to almost all other groups (at least $P<0.05$ ), and differences between subsequent categories of family size were prominent and 
(a)

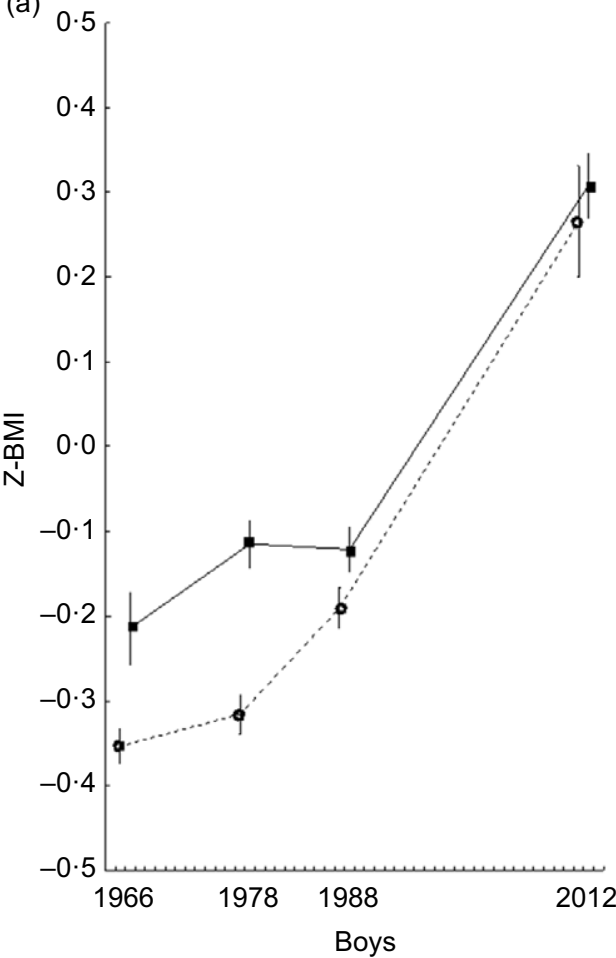

(b)

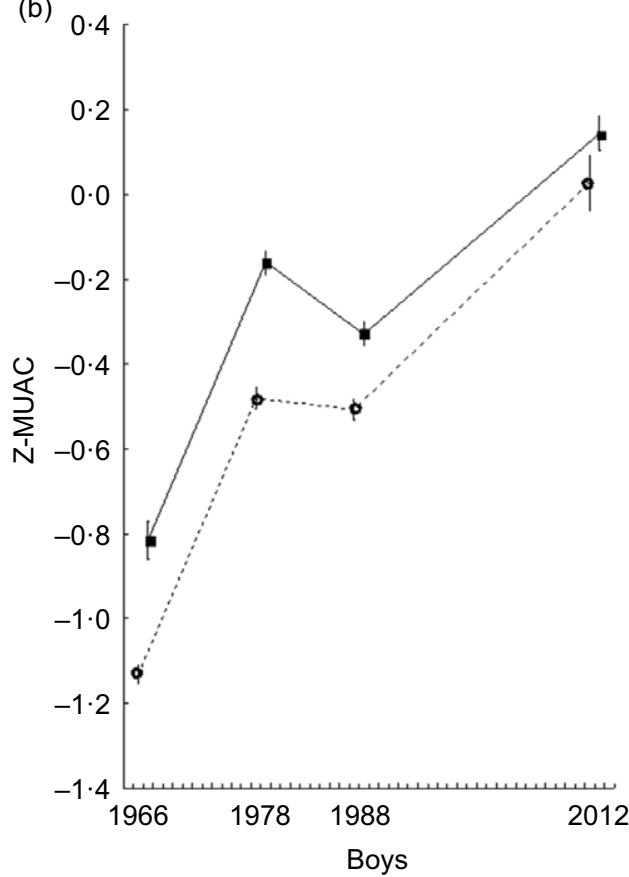

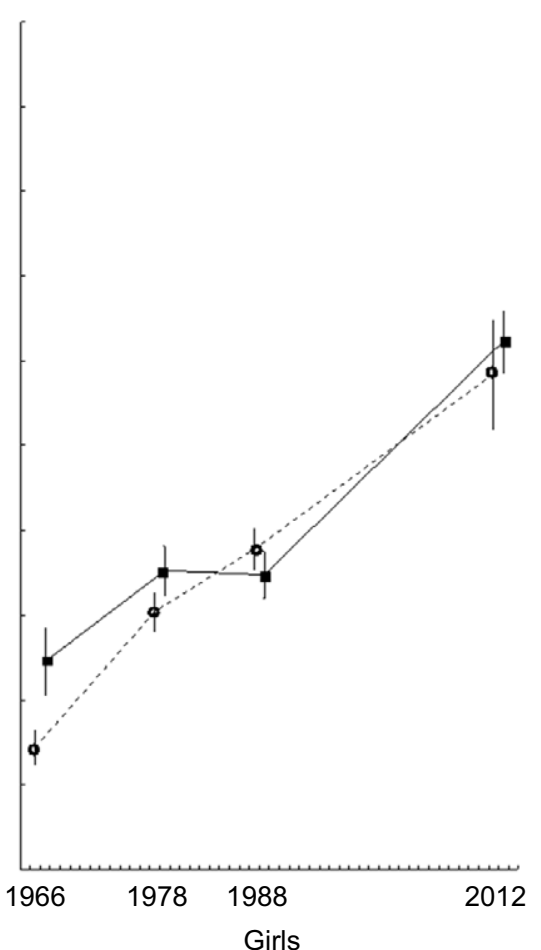

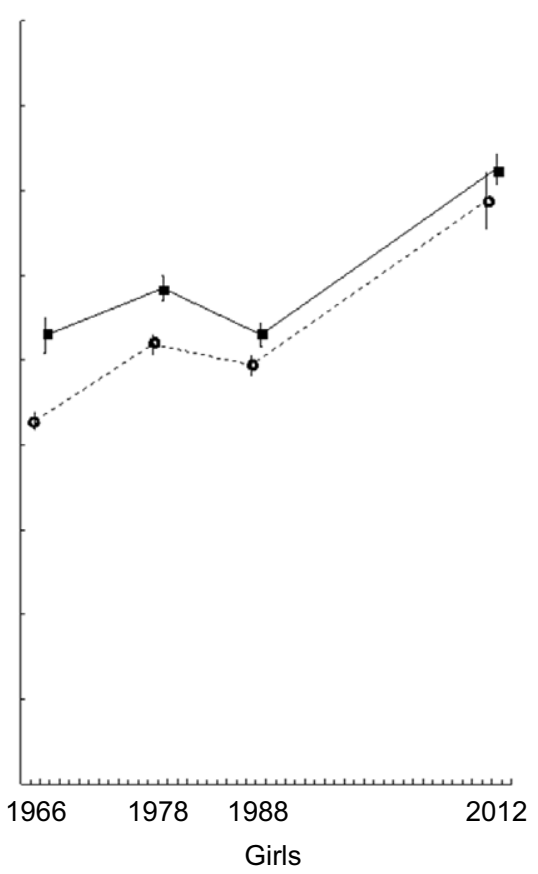

Fig. 4 (a) BMl standardised for age (Z-BMI) and (b) mid-upper arm circumference standardised for age (Z-MUAC) in boys and girls between 1966 and 2012 for different categories of mother's education (vertical lines indicate $95 \% \mathrm{Cl}$ for the mean values). $\overline{.}$. Mother's lower education; ₹, Mother's higher education

significant in most of the Surveys (see Fig. 2 and online supplementary material).

\section{Father's and mother's education}

Within each category of parental education, both $Z$-BMI and $Z$-MUAC increased significantly in boys and girls between 1966 and $2012(P<0.001$; Table 1, Figs 3 and 4 for father's and mother's education, respectively; see online supplementary material). However, the pattern of these changes was different for both measurements. Between 1966 and 1978, Z-BMI significantly increased (at least $P<0.05$ ) only in a half of the cases (see: Fig. 3 and 4; 
compare: Supplementary Material). During the same period, $Z$-MUAC increased significantly in both sexes in each category of parental education (at least $P<0.05$ ). Between 1978 and 1988, no significant changes in Z-BMI were noted among children of parents with higher education, while in children of parents with lower education, significant increases were observed (at least $P<0 \cdot 01$ ). In contrast, between 1978 and 1988, Z-MUAC revealed significant decreases in children of parents with higher education (at least $P<0.01$ ) and no significant changes in both sexes where parents had lower education $(P>0.05)$. Among all children between 1988 and 2012, Z-BMI and Z-MUAC increased significantly in both categories of parental education (at least $P<0 \cdot 01$ ).

With respect to different categories of father's and mother's education in 1966, 1978 and 1988, Z-MUAC revealed significant differences in favour of children with higher parental education more often than Z-BMI (see: Figs 3 and 4; compare online supplementary material). In 2012, both $Z$-BMI and Z-MUAC in boys and girls did not differ significantly with respect to parental education $(P>0.05)$.

\section{Sex differences for Z-BMI and Z-MUAC within socio-economic factors}

With respect to the sex differences for the urbanisation level, $Z$-BMI differed significantly in 2012 in favour of boys from cities, towns and villages $(P<0.001$, almost all other sex differences remained insignificant; Fig. 1). Regarding Z-MUAC, all boys from cities, towns and villages in 1966 and boys from villages in 1978 had significantly lower values of this variable $(P<0.001)$, while in 1988 and 2012, no significant sex differences were observed. Analogously, in 1966, boys from all categories of family size had lower values of $Z$-MUAC compared with girls ( $P<0.001)$, while for $Z$-BMI, no significant sex differences were noted during this Survey $(P>0.05)$. In 2012, $Z$-MUAC did not reveal any sex differences in categories of family size; however, $Z$-BMI was higher in boys than in girls from families with two children $(P<0.001)$. Significant sex differences in both categories of parental education were observed for $Z$-MUAC in 1966 in favour of girls $(P<0.001)$, while $Z$-BMI did not reveal any significant differences between boys and girls during this Survey. There were no significant sex differences for $Z$-MUAC in 2012 among all categories of parental education, while $Z$-BMI was significantly higher in boys from both categories of parental education during the last Survey (at least $P<0.05)$.

\section{Discussion}

The results of this study confirmed our research hypothesis that MUAC is a more sensitive indicator of different SES factors (urbanisation, family size and parental education) compared with BMI. Both measurements showed some social gradients referring to the level of each analysed SES factor; however, $Z$-MUAC revealed more frequently than $Z$-BMI significant differences between categories of particular social factors, especially in 1966, 1978 and 1988. It may be concluded that until the 1990s, Polish society revealed similar socio-economic differences in relative weight and nutritional status as in low-income developing countries up to the same time ${ }^{(14)}$ because children from higher categories of SES factors had significantly higher BMI and MUAC compared with children from lower SES groups. Similar results were obtained in more general analysis of BMI and MUAC within two categories of overall SES in Polish schoolchildren between 1966 and 2012(40). Also, in the present study, between 1978 and 1988, a specific decline in Z-MUAC in different categories of SES factors was observed, reflecting generally worsening living conditions due to the food shortages and price increases in Poland during this time interval (except for children from villages that could have better conditions on rural site ${ }^{(40)}$. However, although MUAC revealed significant intergenerational differences more frequently than BMI in the previous study, both indicators showed similar differences concerning the lower and higher SES category (measured as overall SES). In contrast, more detailed analysis in a current study has indicated significant differences for MUAC more frequently than for BMI referring to the particular categories of SES, such as urbanisation level, family size or parental education.

In 2012, biological differences between children from different levels of urbanisation and particular categories of parental education diminished. The analogous phenomenon of diminishing differences in biological condition between different socio-economic groups was also observed in Sweden in the $1970 \mathrm{~s}^{(41)}$. Also recently in most low- and middle-income countries, especially among women, rapidly increasing values of BMI in rural areas led to the convergence, or even reversal, in its values between cities and villages ${ }^{(18)}$. In our study in 2012, only family size remained a significant factor that still differentiated indicators of relative weight and nutritional status; however, between different categories of family size, more often with respect to $Z$-MUAC than $Z$-BMI.

Concerning the urbanisation level, it may also be observed that $Z$-MUAC revealed significant differences more frequently than $Z$-BMI between cities, towns and villages. Our results are, to some extent, in contrast with the study conducted by Peña Reyes et $a l .{ }^{(11)}$, who observed that in a group of Mexican children between 1970 and 2000, urban-rural differences for an arm circumference were slightly reduced over the time interval compared with greater disparities for relative weight. Nevertheless, children from urban settlements had a generally higher BMI than children from rural areas, similarly as in our study during the years 1966-1978. In 2012, both Z-MUAC and $Z$-BMI did not differ significantly with respect to the 
urbanisation level. Correspondingly, PeytremannBridevaux et al. ${ }^{(16)}$ revealed that in a survey from 2004 involving ten European countries with relatively high GDP (gross domestic product), there were no significant differences in excess weight between urban and rural areas. Although in Poland until the 1990s, there were considerable differences in living conditions between cities and villages, which was visibly reflected in the biological indicators of growth in children ${ }^{(42)}$, after 2000 socioeconomic disparities between these levels of urbanisation significantly diminished, since the living conditions of the rural population significantly improved ${ }^{(43)}$. This socioeconomic equality of living standards in urban and rural areas was reflected in the absence of differences in relative weight and nutritional status of children from cities $v$. villages studied in 2012. However, a recent worldwide review concerning the effect of urbanisation level on excess weight in adults revealed that rural BMI is the main driver of the global obesity epidemic ${ }^{(18)}$. Also, Bac et al. ${ }^{(44)}$ observed that in their survey from 2008 to 2009 conducted in Cracow (Poland) among children 6-13 years of age, overweight was more frequent in boys and girls from rural areas as well as higher percentage of obesity was noted in rural girls, while for boys, obesity was prevailing in urban settings. These results, found in one of the highly developed Polish provinces, might predict a future nationwide trend, already observed in other developed countries ${ }^{(19)}$.

Our results revealed that during the whole period under the study, the family size was the only factor that constantly and significantly affected nutritional and relative weight indicators in children. This association was similar as observed by other researchers: children from families with only one child have the highest relative weight compared with children from larger families ${ }^{(19,20)}$. Probably, this phenomenon is related to food distribution and individual level of resources per person that are often lower in larger families ${ }^{(45)}$. Moreover, our study revealed some significant sex differences in both BMI and MUAC. In 1966, boys, as the more eco-sensitive sex, had significantly lower values of MUAC compared with girls in all categories of family size, while BMI did not reveal any significant sex differences in corresponding groups. Particularly, difficult living conditions of the post-war generation might have been reflected among boys in lower MUAC, implying this indicator as more sensitive to undernutrition than BMI. On the contrary, in 2012, boys revealed no significant differences in MUAC compared with girls, but they had significantly higher BMI. A higher level of BMI in boys was related to a significantly higher level of overall overweight and obesity observed by Gomula et al. in 2012 in boys compared with girls ${ }^{(10,40)}$.

Between 1966 and 1988, parental education significantly differentiated the nutritional and relative weight status of children. In most cases, higher education led to the higher values of MUAC and BMI in offspring. These results are consistent with similar correlations found in developing countries until 1990, where a higher level of education is associated with higher relative weight ${ }^{(14)}$. However, during this time interval, significant differences between particular categories of parental education with respect to anthropometric indicators were more frequently noted for MUAC compared with BMI (see Figs 3 and 4). It is noteworthy that in the most difficult living conditions, in 1966, only MUAC revealed significant sex and educational differences, with its lower values in boys in both categories of parental education. In contrast, in most favourable living conditions in 2012, only BMI showed significant sex differences in favour of boys, but with no difference between parental education categories. It may be concluded that, with respect to sex differences, MUAC was, probably, more sensitive to adverse environmental conditions with limited food resources, while BMI might be more differentiated between boys and girls in more obesogenic environment in Poland in 2012. The lack of differences between particular categories of parental education for both anthropometric indicators in 2012 is to some extent surprising because in better-developed populations, higher-educated people have a higher awareness of healthy lifestyle and dietary habits ${ }^{(46)}$; thus, generally, they have a significantly lower level of excess weight. Correspondingly, other research on Polish schoolchildren conducted in recent years showed that a higher level of parental education is associated with a lower gain in BMI among girls measured longitudinally, from 7 to 18 years of age ${ }^{(47)}$.

Although our study revealed many interesting and significant trends as well as socio-economic differences in BMI and MUAC among the population of Polish children, these Surveys also had some limitations. First, in the last Survey in 2012, the number of participants was lower than that in previous studies, due to the changes in law regulations and, consequently, lower number of written consents from parents or legal guardians of children for participation in this Survey. However, this number was still considerably high ( $n$ 5996) compared with other Polish studies $^{(44,47)}$. Secondly, since the first study protocol was established in 1966 and repeated during the next Surveys, we did not collect any information about physical activity level or dietary habits among children, which could potentially affect relative weight or nutritional status. Nevertheless, the total number of participants was always significantly high and randomly selected in our studies; thus, its variation could compensate for the potentially confounding effect of physical activity or dietary habits. Another limitation of the study was that it was based on the associations between analysed variables derived from the cross-sectional sample, not from the longitudinal data, that are usually more valuable and precise, but also more difficult to collect. It may also be questioned to what extent analysed independent SES factors are substantially reflecting socio-economic conditions of participants. However, before political transformation (1966, 1978 and 1988 
Surveys), differences between particular categories of SES variables were remarkably visible in a Polish population (e.g. due to limited mobility between cities and villages, lower access to higher education and health care among rural inhabitants, or lower income per person in larger families) ${ }^{(48)}$. The 2012 Survey involves population after socio-political transformation, where, for instance, differences in socio-economic conditions between rural and urban residence diminished, but still did not disappear ${ }^{(49)}$

There is a scarcity of data showing how different SES factors affect MUAC, while it is quite well documented with respect to $\mathrm{BMI}^{(32,33,50)}$. Before political transformation and establishing a well-developed form of democracy in Poland, Polish schoolchildren revealed similar SES differences in their relative weight and nutritional status as in other low-income developing populations until the 1990s, where children from urban settlements, living in families with only one child and having parents with higher education, had higher values of relative weight compared with their counterparts from lower socio-economic groups $^{(14)}$. Moreover, the last Polish Anthropological Survey conducted in 2012 showed that urbanisation level and parental education lost their significance in the differentiation of relative weight and nutritional status in children; however, family size remained a significant differentiating social factor. Still, MUAC seems to be a more sensitive nutrition-related indicator of differences in environmental conditions. Although the previous analysis presented by Nowak-Szczepanska et al. ${ }^{(40)}$ revealed that the long-term socio-economic changes differentiated MUAC and BMI in children, the lower and higher categories of overall SES showed mostly similar differences for both anthropometric indicators. The current research contributes to the previous one with detailed analyses, taking into account individual components of SES, indicating that MUAC revealed some particular SES differences more frequently than BMI. Thus, arm circumference might be a more useful anthropometric measure in this context than relative weight. Since nutrition is significantly affected by the SES differences that may lead to social inequalities in health and obesity-related diseases ${ }^{(51)}$, it is particularly important to determine the most reliable anthropometric indicator of nutritional status in population screening.

\section{Acknowledgements}

Acknowledgements: The authors would like to thank all anthropologists, professional staff, participants and other people involved in Polish Anthropological Surveys during the years 1966-2012. Financial support: The survey conducted in 2012 was funded by the National Science Centre in Poland under grant number NN 303804540. The funding organisation did not influence the study. Conflict of interest: None. Authorship: N.N.-S. drafted and edited the manuscript, participated in data collection, prepared database, analysed and interpreted the data. A.G. edited and completed the manuscript for intellectual content, participated in data collection and digitalisation, prepared database and analysed the data. S.K. organised, arranged and coordinated the study conducted in 2012, as well as analysed the data and edited the manuscript for intellectual content. All authors reviewed critically the manuscript. Ethics of buman subject participation: This study was conducted according to the guidelines laid down in the Declaration of Helsinki, and all procedures involving study participants were approved by the Scientific Ethics Committee at the University School of Physical Education in Wroclaw, Poland. Informed consent was obtained from parents of all participants included in the study (in 2012 in a written form, in preceding studies - as oral consent, since Polish law did not demand consent in the written form previously).

\section{Supplementary material}

For supplementary material accompanying this paper visit https://doi.org/10.1017/S1368980020001706

\section{References}

1. Steckel RH (2012) Social and economic effects on growth. In Human Growth and Development, pp. 225-244 [N Cameron \& B Bogin, editors]. San Diego, CA: Academic Press.

2. Tanner JM (1987) Growth as a mirror of the condition of society: secular trends and class distinctions. Pediatr Int 29, 96-103.

3. Schell LM, Burnitz KK \& Gallo MV (2012) Growth as a mirror: is endocrine disruption challenging Tanner's concept? Ann Hum Biol 39, 361-371.

4. Aßmann C \& Hermanussen M (2013) Modeling determinants of growth: evidence for a community-based target in height? Pediatr Res 74, 88-95.

5. Hermanussen M, Alt C, Staub K et al. (2014) The impact of physical connectedness on body height in Swiss conscripts. Anthropol Anz 71, 313-327.

6. Nowak-Szczepanska N, Gomula A, Ipsen MJ et al. (2016) Different effects of living conditions on the variation in BMI and height in children before the onset of puberty. Eur J Clin Nutr 70, 662-666.

7. Chrzanowska M, Koziel S \& Ulijaszek SJ (2007) Changes in BMI and the prevalence of overweight and obesity in children and adolescents in Cracow, Poland, 1971-2000. Econ Hum Biol 5, 370-378.

8. Vignerová J, Humenikova L, Brabec M et al. (2007) Longterm changes in body weight, BMI, and adiposity rebound among children and adolescents in the Czech Republic. Econ Hum Biol 5, 409-425.

9. Mészáros Z, Mészáros J, Völgyi E et al. (2008) Body mass and body fat in Hungarian schoolboys: differences between 1980-2005. J Physiol Anthropol 27, 241-245.

10. Gomula A, Nowak-Szczepanska N, Danel DP et al. (2015) Overweight trends among Polish schoolchildren before and after the transition from communism to capitalism. Econ Hum Biol 19, 246-257. 
11. Peña Reyes M, Tan SK \& Malina RM (2003) Urban-rural contrasts in the growth status of school children in Oaxaca, Mexico. Ann Hum Biol 30, 693-713.

12. Prentice AM (2005) The emerging epidemic of obesity in developing countries. Int J Epidemiol 35, 93-99.

13. Mascie-Taylor CGN \& Goto R (2007) Human variation and body mass index: a review of the universality of BMI cut-offs, gender and urban-rural differences, and secular changes. J Physiol Anthropol 26, 109-112.

14. Monteiro CA, Conde WL, Lu B et al. (2004) Obesity and inequities in health in the developing world. Int J Obes $\mathbf{2 8}$, 1181-1186.

15. Patterson PD, Moore CG, Probst JC et al. (2004) Obesity and physical inactivity in rural America. $J$ Rural Health 20, 151-159.

16. Peytremann-Bridevaux I, Faeh D \& Santos-Eggimann B (2007) Prevalence of overweight and obesity in rural and urban settings of 10 European countries. Prev Med 44, 442-446.

17. Pampel FC, Denney JT \& Krueger PM (2012) Obesity, SES, and economic development: a test of the reversal hypothesis. Soc Sci Med 74, 1073-1081.

18. NCD Risk Factor Collaboration (2019) Rising rural body-mass index is the main driver of the global obesity epidemic in adults. Nature 569, 260-264.

19. Padez C, Mourão I, Moreira P et al. (2005) Prevalence and risk factors for overweight and obesity in Portuguese children. Acta Poediatr 94, 1550-1557.

20. Chen AY \& Escarce JJ (2010) Family structure and childhood obesity, early childhood longitudinal study - kindergarten cohort. Prev Chronic Dis 7, A50.

21. Golan M (2006) Parents as agents of change in childhood obesity - from research to practice. Int J Pediatr Obes $\mathbf{1}$, 66-76.

22. Lamerz A, Kuepper-Nybelen J, Wehle C et al. (2005) Social class, parental education, and obesity prevalence in a study of six-year-old children in Germany. Int J Obes 29, 373-380.

23. Frost MB, Forste R \& Haas DW (2005) Maternal education and child nutritional status in Bolivia: finding the links. Soc Sci Med 60, 395-407.

24. Ng M, Fleming T, Robinson M et al. (2014) Global, regional, and national prevalence of overweight and obesity in children and adults during 1980-2013: a systematic analysis for the Global Burden of Disease Study 2013. Lancet 384, 766-781.

25. Peterson CM, Su H, Thomas DM et al. (2017) Tri-ponderal mass index vs body mass index in estimating body fat during adolescence. JAMA Pediatr 171, 629-636.

26. Chomtho S, Fewtrell MS, Jaffe A et al. (2006) Evaluation of arm anthropometry for assessing pediatric body composition: evidence from healthy and sick children. Pediatr Res 59, 860-865.

27. Freedman DS \& Sherry B (2009) The validity of BMI as an indicator of body fatness and risk among children. Pediatrics 124, S23-S34.

28. Briend A, Alvarez JL, Avril N et al. (2016) Low mid-upper arm circumference identifies children with a high risk of death who should be the priority target for treatment. BMC Nutr 2, 63.

29. Craig E, Bland R, Ndirangu J et al. (2014) Use of mid-upper arm circumference for determining overweight and overfatness in children and adolescents. Arch Dis Child 99, $763-766$.

30. Chaput JP, Katzmarzyk PT, Barnes JD et al. (2017) Mid-upper arm circumference as a screening tool for identifying children with obesity: a 12-country study. Pediatr Obes 12, 439-445.

31. Mazıcıŏglu MM, Hatipoğlu N, Öztürk A et al. (2010) Waist circumference and mid-upper arm circumference in evaluation of obesity in children aged between 6 and 17 years. J Clin Res Pediatr Endocrinol 2, 144-150.
32. Kozieł S, Szklarska A, Bielicki T et al. (2006) Changes in the BMI of Polish conscripts between 1965 and 2001: secular and socio-occupational variation. Int J Obes 30, 1382-1388.

33. Neuman M, Kawachi I, Gortmaker S et al. (2013) Urban-rural differences in BMI in low-and middle-income countries: the role of socioeconomic status. Am J Clin Nutr 97, 428-436.

34. Hulanicka B \& Waliszko A (1991) Deceleration of age at menarche in Poland. Ann Hum Biol 18, 507-513.

35. Martin R \& Saller K (1957) Textbook of Anthropology in a Systematic Presentation With Special Attention to Anthropological Methods, Volume 1 (in German). Stuttgart: Gustav Fischer.

36. Cole TJ, Bellizzi MC, Flegal KM et al. (2000) Establishing a standard definition for child overweight and obesity worldwide: international survey. BMJ 320, 1240.

37. Mramba L, Ngari M, Mwangome M et al. (2017) A growth reference for mid upper arm circumference for age among school age children and adolescents, and validation for mortality: growth curve construction and longitudinal cohort study. BMJ 358, j3423.

38. Cole TJ (1990) The LMS method for constructing normalized growth standards. Eur J Clin Nutr 44, 45-60.

39. Dell Inc (2016) Dell Statistica (data analysis software system), version 13. Available at software.dell.com (accessed June 2016).

40. Nowak-Szczepanska N, Gomula A \& Koziel S (2019) Midupper arm circumference and body mass index as different screening tools of nutritional and weight status in Polish schoolchildren across socio-political changes. Sci Rep 9, 1-7.

41. Lindgren G (1976) Height, weight and menarche in Swedish urban school children in relation to socio-economic and regional factors. Ann Hum Biol 3, 501-528.

42. Hulanicka B, Brajczewski C, Jedlinska W et al. (1990) CityTown-Village: Growth of Children in Poland in 1988. Wroclaw: Monographs of the Institute of Anthropology, Polish Academy of Sciences.

43. GUS - Central Statistical Office, Social Surveys and Living Conditions Department (2017) Socio-Economic Situation of Households in 2000-2015: Urban-Rural Diversity. Warsaw: Statistical Information and Elaborations.

44. Bac A, Woźniacka R, Matusik S et al. (2012) Prevalence of overweight and obesity in children aged 6-13 yearsalarming increase in obesity in Cracow, Poland. Eur $J$ Pediatr 171, 245-251.

45. Bielicki T, Szklarska A, Kozieł S et al. (2003) Political Transformation in Poland in the Context of Anthropological Studies of 19-Year-Old Men. Wrocław: Monographs of Institute of Antropology, Polish Academy of Sciences.

46. Monteiro CA, Conde WL \& Popkin BM (2001) Independent effects of income and education on the risk of obesity in the Brazilian adult population. J Nutr 131, 881S-886S.

47. Wronka I (2014) Socioeconomic status, body mass index and prevalence of underweight and overweight among Polish girls aged 7-18: a longitudinal study. J Biosoc Sci 46, 449-461.

48. Bielicki T \& Szklarska A (1999) The stratifying force of family size, urbanization and parental education in socialist-era Poland. J Biosoc Sci 31, 525-536.

49. Gomula A \& Koziel S (2018) Secular trend and social variation in age at menarche among Polish schoolgirls before and after the political transformation. Am J Hum Biol 30, e23048.

50. van Stralen MM, te Velde SJ, van Nassau F et al. (2012) Weight status of European preschool children and associations with family demographics and energy balance-related behaviours: a pooled analysis of six European studies. Obes Rev 13(S1), 29-41.

51. Alkerwi A, Vernier C, Sauvageot N et al. (2015) Demographic and socioeconomic disparity in nutrition: application of a novel Correlated Component Regression approach. BMJ Open 5, e006814. 